\title{
Obtención de materiales con comportamiento tixotrópico mediante
}

\section{"compocasting"}

\author{
S. Alcantara*, C. Merizalde*, J.M. Cabrera, ${ }^{* * * *}$ y J.M. Prado,,*** \\ Resumen El comportamiento tixotrópico de un material está asociado a la coexistencia de fases \\ líquida y sólida en las proporciones adecuadas para que la mezcla no se desmorone. En estas \\ condiciones, la mezcla es relativamente insensible a esfuerzos de cizalladura pudiendo \\ deformarse muy fácilmente. En este trabajo, se plantea la elaboración de un material \\ tixotrópico a partir de un compuesto, formado por una aleación 63 \% Sn-37 \% Pb (que \\ actuará como el agente ligante o fase líquida), al que se añade un porcentaje determinado \\ de partículas de cobre (que actúan de fase sólida). Para ello, se realiza la mezcla de las \\ partículas de cobre con la aleación $\mathrm{Sn}$-Pb en estado líquido a $250^{\circ} \mathrm{C}$ por medio de agitación \\ mecánica. El trabajo comprendió la preparación de diversos porcentajes de mezcla, en un \\ intervalo entre 10 y $50 \%$ en peso de cobre (de morfología tanto esférica como irregular). \\ El material así obtenido se caracterizó por medio de microscopía óptica y electrónica de \\ barrido.
}

Palabras clave Tixotropía. Compuesto intermetálico. Aleación eutéctica. Aleaciones reforzadas con partículas.

\section{Preparation of thixotropic materials by "compocasting"}

\begin{abstract}
The thixotropic behavior of a material is associated to the coexistence of a liquid and a solid phase in suitable proportions so that the mixture does not crumble. In these conditions, the slurry is relatively insensible to shear stresses, being able to deform itself very easily. In this work, a thixotropic material formed by an alloy $63 \% \mathrm{Sn}-37 \% \mathrm{~Pb}$ (which acts as the union agent or liquid phase), and a determined percentage of copper particles (which act as the solid phase) will be elaborated. This mixture is made by means of mechanical agitation at $250^{\circ} \mathrm{C}$ when the $\mathrm{Sn}-\mathrm{Pb}$ alloy is in the liquid state. The work includes the preparation of slurries with different percentages of $\mathrm{Cu}$ in the interval from 10 to $50 \%$ in weight (the $\mathrm{Cu}$ particles being both spherical and irregular morphology). The material obtained was characterized by optical and Scanning Electron Microscope.
\end{abstract}

Keywords

Thixotropy. Intermetallic compound. Eutectic alloys. Particle-reinforced alloys.

\section{INTRODUCCIÓN}

El procesamiento de metales semi-sólidos es un método relativamente nuevo, usado para la transformación de aleaciones en condición semi-sólida a productos conformados o parcialmente acabados. En los inicios, el interés en las propiedades mecánicas y reológicas de estas aleaciones, que eventualmente llevaron a su procesamiento, puede otorgarse a los trabajos de Flemings et al. ${ }^{[1]}$, a prin- cipios de la década de los 70. Sus trabajos estaban dirigidos originalmente al problema de agrietamiento en caliente en aleaciones fundidas, pero se descubrió una nueva tecnología para el semiacabado de formas complejas. De igual manera, Spencer et al. ${ }^{[2]}$, descubrieron las particulares e inusuales propiedades reológicas de una mezcla $\mathrm{Sn}$ - $\mathrm{Pb}$ fuertemente agitada. Si se observa el comportamiento de mezclas semi-sólidas en las cuales los sólidos existen en forma de partículas esféricas o en forma de

$\left({ }^{*}\right)$ Departamento de Ciencia de los Materiales e Ingeniería Metalúrgica, ETSEIB-Universitat Politécnica de Catalunya, Av. Diagonal 647, 08028Barcelona, España.

${ }^{* *}$ Centre Tecnològic de Manresa, Av. de les Bases de Manresa 1, 08240 - Manresa, España. 
estructuras globulares se ve que si estas mezclas permanecen sin perturbación, se endurecen y pueden comportarse como un sólido, pero fluyen como líquido cuando están sometidas a esfuerzos cizallantes ${ }^{[3-5]}$. Así, los lingotes de aleaciones semisólidas pueden ser inyectados en moldes o forjados en matrices cerradas para producir componentes con buen acabado superficial, libre de porosidades y que presenten microestructuras finas y uniformes, que pueden, además, ser tratadas térmicamente para mejorar sus propiedades mecánicas ${ }^{[3,4,6 \text { y } 7]} \cdot \mathrm{Pa}$ ra la obtención de piezas a partir de metal en estado semisólido, es necesario que la materia prima presente una microestructura esférica y de grano fino. Los primeros estudios realizados en este tema ${ }^{[1-3}$ y 5$]$, mostraron que una manera simple y efectiva de conseguirlo consistía en agitar mecánicamente la aleación durante el proceso de solidificación. Estos estudios han permitido adicionalmente conocer una serie de parámetros clave. En primer lugar, el diámetro de las partículas sólidas depende, primordialmente, de la velocidad de solidificación y, en segundo lugar, la optimización de microestructuras esferoidales se controla mediante el grado de agitación. Así, con altas velocidades de solidificación se obtienen diámetros de partículas menores, y con elevados grados de agitación se consiguen partículas más esféricas y menos aglomeraciones.

La gran ventaja de este sistema de agitación reside en su simplicidad, lo que posibilita la realización de ensayos de una forma rápida y económica, y su principal inconveniente es la facilidad con la que el material de ensayo se contamina e incorpora óxidos y otras impurezas.

\section{MATERIALES Y PROCEDIMIENTO EXPERIMEN- TAL}

En el presente trabajo se pretende tener, por un lado, un metal con bajo punto de fusión al que se elevará a estado líquido mediante la aplicación de calor. A este material fundido se le añadirá otro metal con un punto de fusión mayor y que no llegará a fundir. Este segundo material, que estará en forma de polvo, será el que proporcione el porcentaje de fase sólida. Así, el metal fundente tendrá la función de actuar como matriz de la aleación en la que quedarán atrapadas las partículas de polvo metálico, que actuarán como partículas de refuerzo.

La mezcla está compuesta de una aleación eutéctica de estaño-plomo y de partículas de cobre puro con dos morfologías diferentes: esféricas e irregulares, ambas con partículas de $100 \mu \mathrm{m}$ aproximadamente. La aleación eutéctica contiene 61,9 $\%$ en peso de estaño y $38,1 \%$ en peso de plomo o en porcentaje atómico, un 63 y $37 \%$, respectivamente. Su temperatura de fusión es de $183^{\circ} \mathrm{C}$ (ver Fig.1). El eutéctico así obtenido presenta una microestructura laminar o de placas compuesta de fases $\alpha$ y $\beta$. Se ha elegido esta aleación ya que tiene una baja temperatura de fusión lo que facilita, en primer lugar, la preparación del material y, en segundo, la posterior utilización del material tixotrópico obtenido. Otra razón es la pequeña diferencia de densidad entre las fases sólida y líquida, lo que reduce considerablemente la posibilidad de que se produzca segregación o flotación de partículas.

Para que se mezclen bien los elementos se debe poner una pasta decapante para soldaduras de estaño. Esta pasta es especial para, por capilaridad, soldar todos los metales excepto el aluminio. Actúa como decapante, eliminando el óxido que rodea las partículas de cobre y creando una buena cohesión de las partículas con la matriz. Para la producción de las muestras tixotropicas, se utilizó un horno de crisol, dotado de un agitador mecánico. Una vez colocados los materiales en el horno y alcanzada la temperatura adecuada se agitaba por un tiempo determinado, a una velocidad inicial de $190 \mathrm{rpm}$.

Esta velocidad no debe ser muy alta para que la mezcla no se quede en las paredes del horno, pero tampoco debe ser demasiado lenta para que no se aglomeren las partículas de cobre. A continuación, se colocan $30 \mathrm{~g}$ de pasta por cada $10 \%$ en peso de cobre. Inmediatamente después, se añaden las partículas de cobre en frío, en flujo

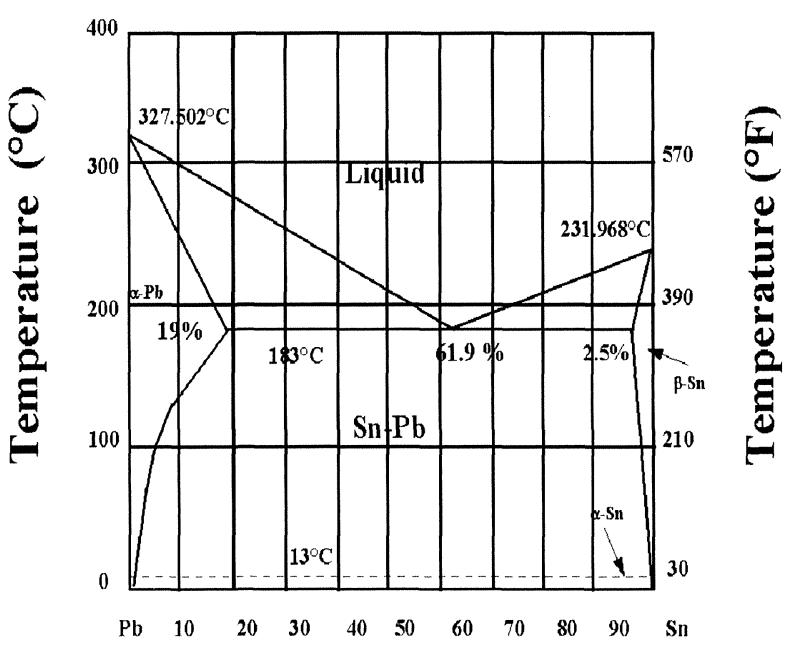

Figura 1. Diagrama de fase de la aleación estaño-plomo.

Figure 1. Tin-lead alloy phase diagram. 
continuo en la aleación bañada en la pasta y con agitación.

\subsection{Preparación de la mezcla con partículas de cobre irregulares}

Se siguieron dos caminos diferentes de preparación de probetas. Uno para las mezclas de $10 \%$ y $20 \%$ en peso de cobre y otro para las mezclas con un porcentaje superior a $20 \%$; el primer modo de preparación de las probetas no se puede utilizar para preparar las últimas por que la mezcla obtenida es demasiado "espesa" y muy difícil de vaciar.

\subsection{Preparación de la mezcla con partículas de cobre esféricas}

La mezcla se obtiene de manera idéntica al caso de partículas irregulares, así como la elaboración de las probetas. De igual forma, la obtención de las probetas es válida para porcentajes no muy elevados de cobre (20 y $35 \%$ ), pues la parte líquida de la mezcla es suficiente como para facilitar la fluencia de la esta y, por tanto, el llenado del molde. Para porcentajes superiores (50\% por ejemplo) surgieron grandes inconvenientes a la hora de vaciar y manipular la mezcla obtenida.

\section{RESULTADOS Y DISCUSIÓN}

\subsection{Partículas irregulares}

Empleando partículas irregulares se prepararon mezclas de $10,20,30,32,35$ y $40 \%$ en peso de cobre. Las mezclas de 10 a $30 \%$ presentaron un aspecto líquido lejos del comportamiento tixotrópico. En cambio, las muestras de 32 y $35 \%$ mostraron inicialmente dicho comportamiento aunque a medida que transcurría la agitación, la mezcla se volvía muy "arenosa" y perdía las características tixotrópicas. Ese aspecto arenoso se mostró enseguida con la composición de $40 \%$. En la muestra con $10 \%$ de cobre de partículas irregulares se observó claramente una estructura laminar (zona 1 de la figura 2) eutéctica de estaño plomo $(\beta$ y $\alpha)$ junto con zonas de eutéctico globular. En algunos casos, se apreciaron islas de plomo con pequeñas partículas de estaño en su interior (zona 2 de la figura 2). De igual manera, se observó que alrededor de las partículas de cobre aparece una fase que tiene una morfología de "pétalos" (Fig. 3) ${ }^{[8]}$. Esta fase, según la bibliografía ${ }^{[8-10]}$, es un intermetálico estaño-cobre. Esta fase intermedia muestra

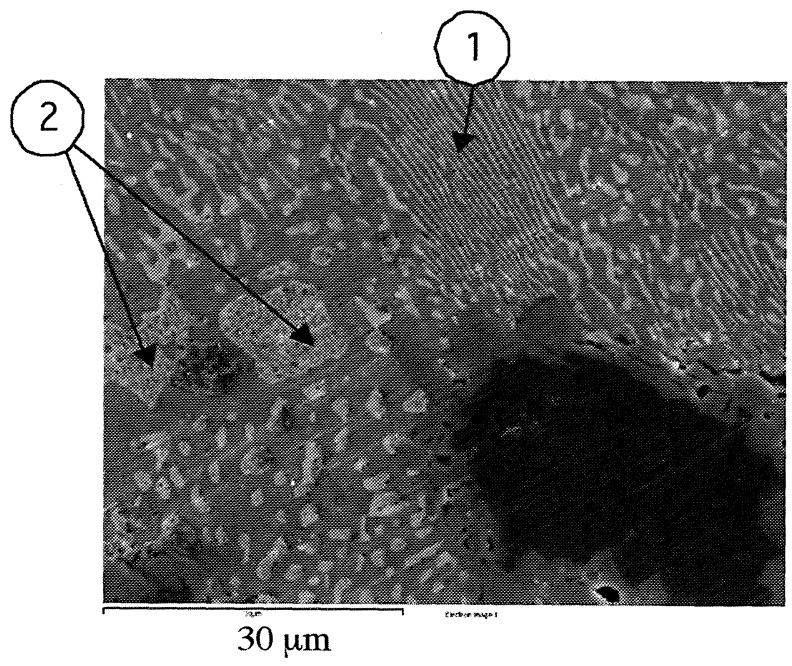

Figura 2. Matriz $\mathrm{Sn}-\mathrm{Pb}$.

Figure 2. Sn-Pb Matrix.

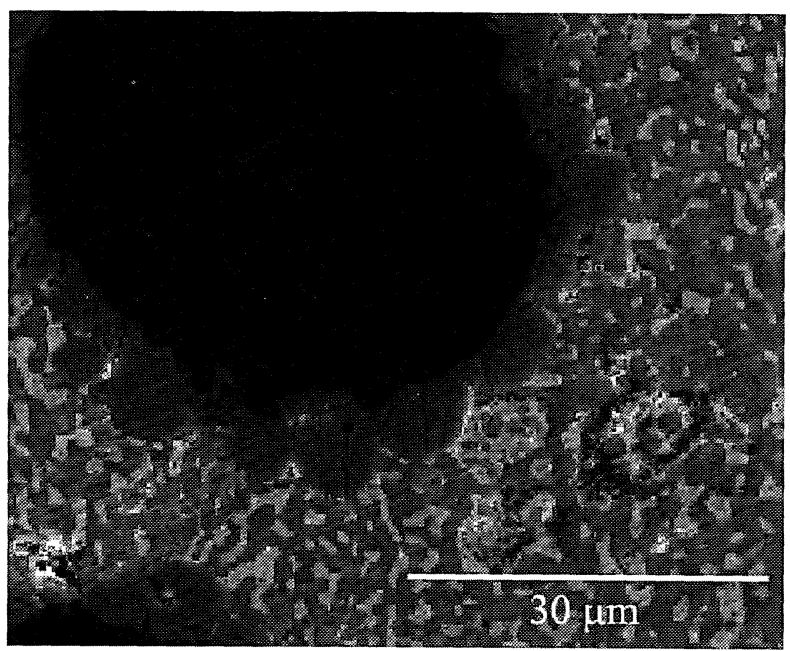

Figura 3. Compuesto intermetálico.

Figure 3. Intermetallic compound.

por una parte que hay interacción entre el cobre y el estaño y, por otra parte, permite la buena cohesión de las partículas de cobre dentro la matriz de estaño-plomo. En efecto, entre el cobre y una aleación eutéctica de estaño-plomo se suelen formar sólo dos fases intermetálicas, a saber: $\mathrm{Cu}_{3} \mathrm{Sn}$ y $\mathrm{Cu}_{6} \mathrm{Sn}_{5}$, siendo el último el que tiene una morfología de conchas ("scallop") o de pétalos como ha sido observado en las mezclas preparadas. Así, se puede decir que la fase observada es la fase intermetálica $\mathrm{Cu}_{6} \mathrm{Sn}_{5}$. En la mezcla con $20 \%$ de cobre apenas se aprecia estructura laminar, estando compuesta de estaño $\alpha$ y de plomo $\beta$ y mucha zonas hipoeutécticas, ricas en plomo, con partículas de estaño, lo que se explica por la mayor cantidad de partículas de cobre. En la mezcla con $30 \%$ de 
cobre se observó morfología eutéctica laminar combinada con zonas hipoeutécticas. La última está, lógicamente, cerca de las zonas donde hay más partículas de cobre (zona 1 de la figura 4).

En las mezclas con $32 \%$ de cobre lo primero que se observa es que hay muchas aglomeraciones de plomo. La mezcla no tiene una estructura eutéctica sino una estructura hipoeutéctica del diagrama de estaño-plomo (Fig. 5). Asimismo las partículas de cobre no tienen buena cohesión en algunos sitios con la matriz apreciándose grietas en la fase intermetálica así como decohesión entre la partícula de cobre y la fase intermetálica.

Por lo que hace referencia a las mezclas con $35 \%$ de $\mathrm{Cu}$ (irregulares) las aglomeraciones de

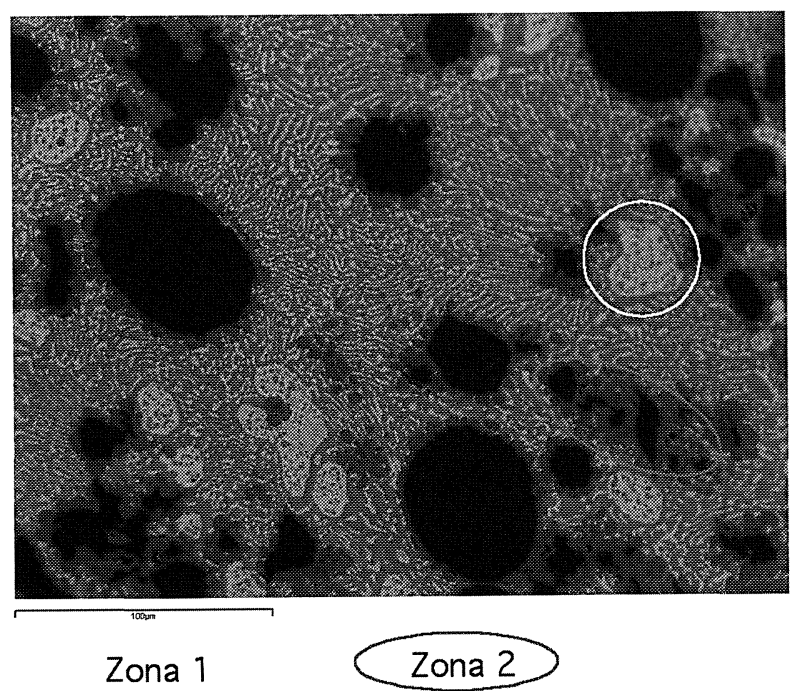

Figura 4. Mezcla con $30 \%$ de cobre.

Figure 4. Slurry with $30 \%$ of copper.

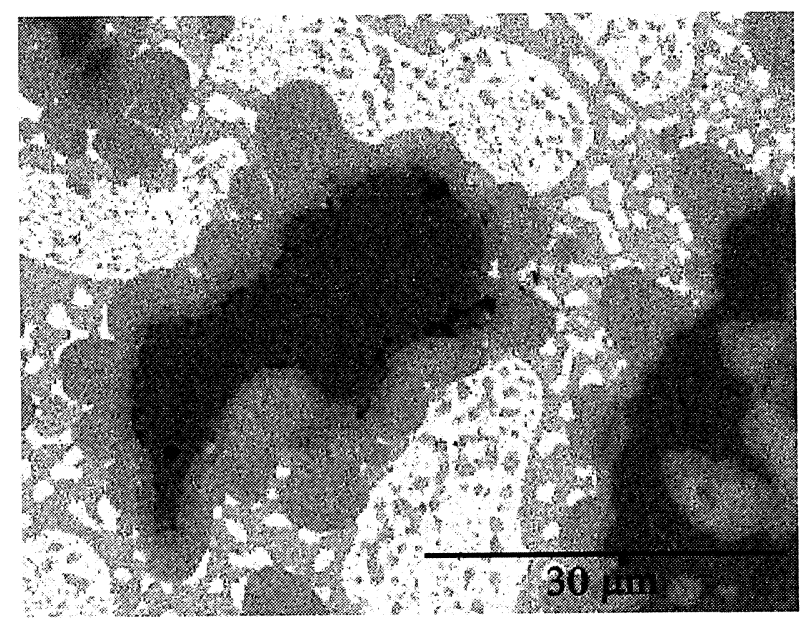

Figura 5. Estructura hipoeutéctica de la mezcla de $32 \%$ cobre.

Figure 5. Hypoeutectic structure of the slurry of $32 \% \mathrm{Cu}$. plomo son superiores a las de la mezcla de $32 \%$ de cobre (Fig. 6).Por lo que hace referencia a las mezclas con $40 \%$ de cobre se observa (Fig. 7) que las partículas de cobre no se han adherido perfectamente a la matriz y que están rodeadas de poros. Además, no todas las partículas de cobre están rodeadas de la fase intermetálica, muy posiblemente porque no hay suficiente estaño para facilitar la reacción, al haber tanto cobre.

\subsection{Partículas esféricas}

Empleando partículas esféricas se prepararon mezclas de 20, 35 y $50 \%$ en peso de cobre. Únicamente la mezcla de $50 \%$ presentó características tixotrópicas, si bien es cierto que tras tiempos de agitación muy largos (más de $30 \mathrm{~min}$ ) la mezcla

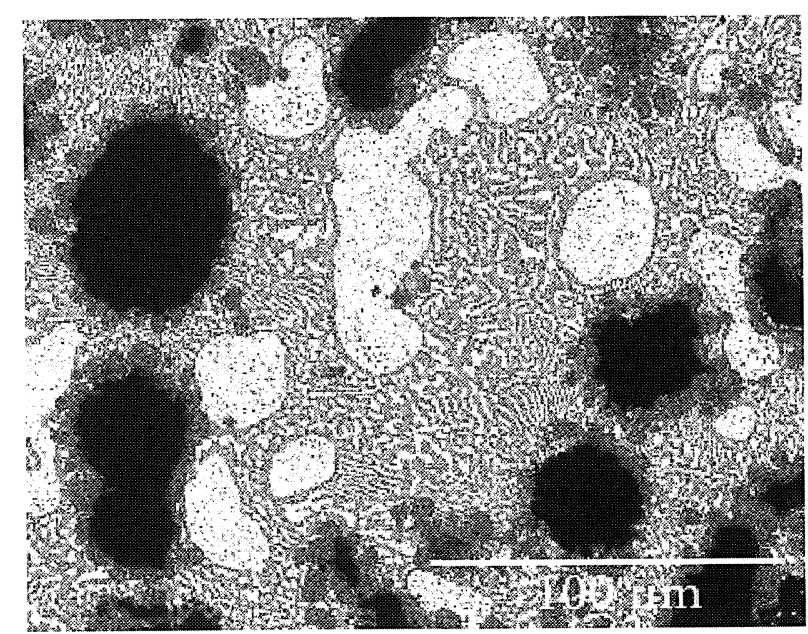

Figura 6. Aglomeraciones de plomo en una mezcla con $35 \%$ de Cu.

Figure 6. Lead agglomerations in a slurry with $35 \%$ of $\mathrm{Cu}$.

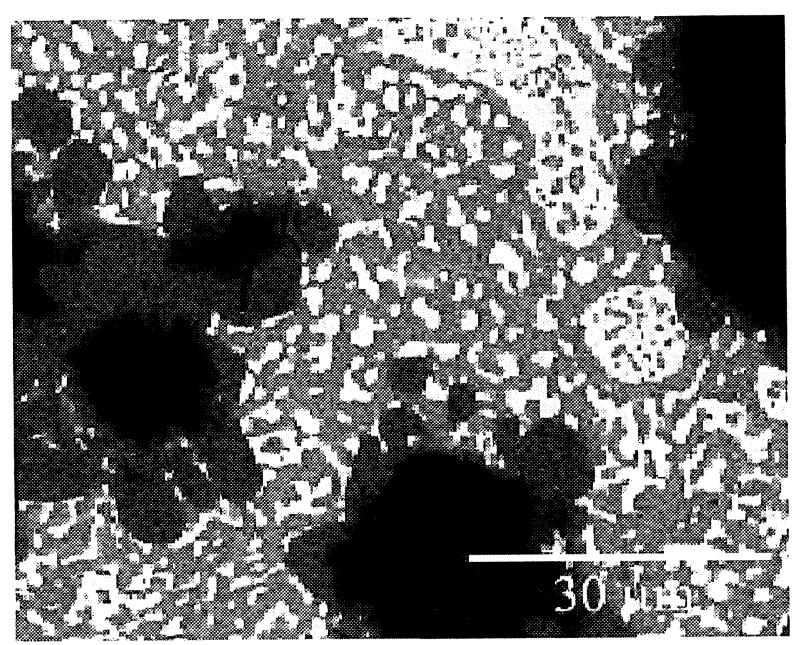

Figura 7. Grietas en el intermetálico.

Figure 7. Intermetallic's cracks.

Rev. Metal. Madrid Vol. Extr. (2005) 111-115 
adquirió un aspecto arenoso. De manera semejante al análisis realizado con las partículas irregulares. Para las composiciones de 20 y $35 \%$ de cobre se observa una desordenada distribución de dichas partículas mientras que para la de $50 \%$ la distribución es bastante uniforme (Fig. 8), las estructuras formadas con las partículas esféricas son muy similares a las observadas para partículas irregulares, pero presentan una mejor adhesión partículamatriz.

\section{CONCLUSIONES}

En el caso de la aleación $\mathrm{Sn}-\mathrm{Pb}$ reforzada con partículas irregulares de cobre se obtuvo un material en estado semi-sólido para una mezcla con $32 \%$ de cobre; sin embargo, este material no presento un comportamiento claramente tixotropico. La mezcla nada tiene que ver con el comportamiento obtenido con partículas de cobre de morfología esférica, la cual si presento un comportamiento tixotropico a $50 \%$ de cobre, por lo que se puede concluir que las partículas esféricas favorecen el comportamiento tixotropico de los materiales, mientras las partículas irregulares son perjudiciales.

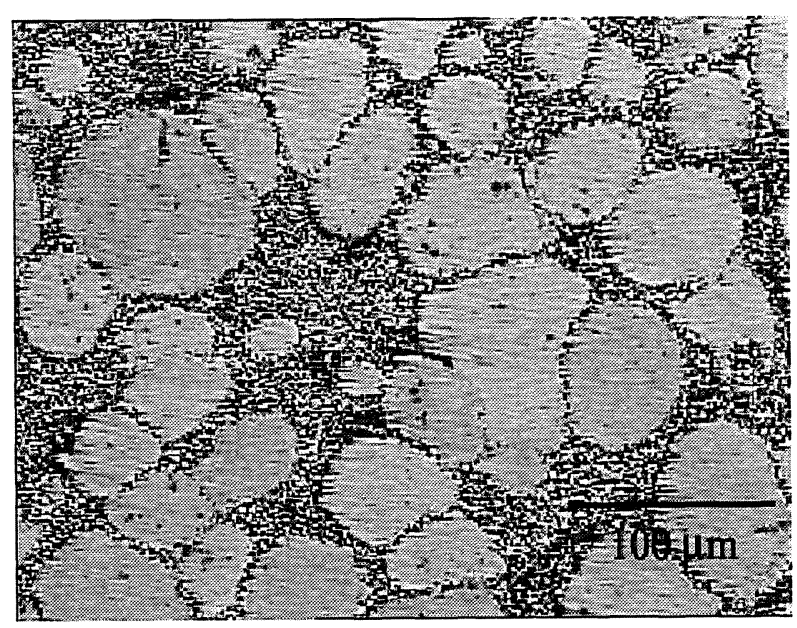

Figura 8. Mezcla tixotrópica, 50 \% Cu.

Figure 8. Thixotropic slurry, $50 \% \mathrm{Cu}$.
De igual manera, este proyecto ha permitido comprender la importancia de la fase intermetálica $\mathrm{Cu}_{6} \mathrm{Sn}_{5}$ que se forma entre las partículas de cobre y la matriz. En efecto, la microestructura de la mezcla está compuesta de una matriz de estaño-plomo con partículas de cobre adheridas a ella por una fase intermetálica $\mathrm{Cu}_{6} \mathrm{Sn}_{5}$ de morfología de pétalos ("scallops") que se forman a su alrededor al reaccionar con el estaño de la matriz. Esta fase aumenta cuando aumenta el porcentaje de cobre para formar una sola fase entre las partículas de cobre cercanas. Como el estaño reacciona más con el cobre y la energía de superficie del plomo es más baja que la del estaño, se forman regiones hipoeutécticas en la matriz, es decir, regiones ricas en plomo, que devienen más numerosas cuando aumenta el porcentaje de cobre en la mezcla.

Además, la fase intermetálica $\mathrm{Cu}_{6} \mathrm{Sn}_{5}$ evoluciona con el tiempo y la temperatura, lo que tendrá su importancia en la utilización posterior del material, por lo que se hacen necesarios unos estudios más profundos de la formación y de la evolución de esta fase.

\section{REFERENCIAS}

[1] M.C. Flemmings, Metall. Trans. A 22 (1991) 957-981.

[2] D.P. Spencer, R. Meehrabian and M.C. Flemmings, Metall. Trans. A 3 (1972) 1.925-1.932.

[3] D.H. Kirkwood, Int. Mater. Rev. 39 (1994) 173-189.

[4] M. Da Silva y J.M. RodríGuez-Isabe, Rev. Metal. Madrid 39 (2003) 452-460.

[5] P.A. JOly y R. Meehrabian, J. Mater. Sci. 11 (1976) 1.393-1.418.

[6] W. LAPKOWSKI y J. SinCZAK, Metall. Foundry Eng. 27 (2001) 97-103.

[7] C.S. Rice y P.F. Mendes, Adv. Mater. \& Procces. (2001) 49-52.

[8] W.K. ChOI, S.Y. Jang, J.H. Kim, K.W. PAIK y H.M. LeE, J. Mater. Res. 17 (2002) 597-599.

[9] K.S. BaE y S.J. KIM CHOI, J. Mater. Res. 17 (2002) 743 746.

[10] T.Y. LeE, W.J. Chol y K.N. Tu, J. Mater. Res. 17 (2002) 291-301. 